\title{
On Linear Stability Analysis of High-Order Finite-Difference Methods
}

\author{
David W. Zingg,* and Matthew Lederle ${ }^{\dagger}$ \\ Institute for Aerospace Studies, University of Toronto \\ 4925 Dufferin St., Toronto, Ontario M3H 5T6, Canada
}

\begin{abstract}
In order to produce the desired global order of accuracy, high-order finite-difference methods require suitably accurate stable boundary schemes. Various tools can be helpful in providing insight into the stability of numerical boundary schemes, including spectra, pseudospectra, and the singular value decomposition. In this paper, these tools are applied to several different discretizations of the linear convection equation which display various types of instability. Pseudospectra are seen to be a convenient and effective means of detecting instabilities. The same instabilities can also be revealed by either the spectrum or the spectrum of the associated circulant matrix resulting from the assumption of periodic boundary conditions. Finally, the singular value decomposition is shown to be a good indicator of the cause of the instability.
\end{abstract}

\section{Introduction}

One of the challenges in the development of high-order finite-difference discretizations is the need for stable boundary schemes of sufficient accuracy. For continuous hyperbolic problems, $N$ th-order global spatial accuracy is achieved when the interior scheme is order $N$ and the boundary schemes are of order $(N-1)$ or better. ${ }^{1}$ If the boundary schemes are of order lower than $(N-1)$, then the benefit of the high-order interior scheme can be greatly reduced.

The challenge of developing boundary schemes for high-order finite-difference methods is compounded by the complexity of proving Lax-Richtmyer stability for hyperbolic initial-boundary-value problems. Application of the normal mode analysis of Godunov and Ryabenkiii ${ }^{2}$ and Gustafsson, Kreiss, and Sundstrom $(\mathrm{GKS})^{3}$ to general high-order difference methods can be exceedingly difficult. ${ }^{4}$

Carpenter et al. ${ }^{5}$ and Zingg and Lomax ${ }^{6}$ developed stable boundary schemes for sixth-order compact and noncompact interior schemes, respectively. These boundary operators were found in an ad hoc manner. Subsequently, efforts have concentrated on the development of more systematic approaches to the development of stable boundary schemes using summation-by-parts energy norms ${ }^{5,7-10}$ and the simultaneous approximation term method. ${ }^{11}$

Asymptotic stability is not a sufficient condition for Lax-Richtmyer stability. In other words, a semidiscrete finite-difference operator with eigenvalues within the stability region of the time integration method is not necessarily stable; neither is a fully-discrete operator with eigenvalues within the unit circle. For nonnormal matrices, the eigenvalue spectrum, while providing an accurate picture of the long-time asymptotic behaviour, does not accurately describe the solution behaviour at finite time. For such matrices (and operators) the pseudospectrum can provide a much more accurate picture. Reddy and Trefethen ${ }^{12}$ applied pseudospectra to the stability analysis of finite-difference methods. The ability of pseudospectra to reveal GKS instabilities is discussed in the recent book by Trefethen and Embree. ${ }^{13}$ The authors remark that the connection between pseudospectra and the stability of numerical boundary schemes has gone largely unexploited; the sole published exception is a 1997 paper by Zingg. ${ }^{14}$

This paper is something of a sequel to the 1997 paper by the first author. That paper presented a conjecture that can be restated as the following question: Can a method be Cauchy stable (i.e. stable if

*Professor, Senior Canada Research Chair in Computational Aerodynamics, 2004 Guggenheim Fellow, Senior AIAA Member.

†Undergraduate Student 
periodic boundary conditions are assumed) and asymptotically stable, but not Lax-Richtmyer stable? The usefulness of the singular value decomposition in providing insight into instabilities was also demonstrated. The objective of the present paper is to explore these issues further, particularly in light of the examples given by Trefethen and Embree ${ }^{13}$ showing the effectiveness of pseudoeigenvalues in revealing the existence of unstable modes.

In the next section, the notation and stability definitions are introduced, and pseudospectra are defined. This is followed by five examples showing various types of instability, beginning with an instability associated with the numerical scheme at the inflow boundary. The second example gives stable inflow boundary operators, while the third example is a mild instability associated with the interior scheme. Examples

four and five are unstable cases from Trefethen and Embree. ${ }^{13}$ The first is an explicit scheme, the second is implicit. In both cases the instability is associated with the outflow boundary scheme.

\section{Background}

\section{A. Model Problem and Notation}

We consider the following initial-boundary-value problem (the linear convection equation):

$$
\frac{\partial U}{\partial t}+\frac{\partial U}{\partial x}=0
$$

on the domain $0 \leq x \leq 1$. The initial condition is $U(x, 0)=U_{0}(x)$. This equation governs the propagation of a scalar, $U(x, t)$, to the right with unit speed. The left boundary is called the inflow boundary, since in general a time-varying boundary condition can be specified there, representing an incoming wave. In the study of stability, we need only consider the Dirichlet boundary condition $U(0, t)=0$. The right boundary is called the outflow boundary. The wave leaves the domain through this boundary without reflection. This equation is a suitable representative model problem for hyperbolic systems.

The notation here parallels that in Lomax et al. ${ }^{15}$ The numerical approximation to $U(x, t)$ is written as

$$
u_{j}^{n} \approx U(j \Delta x, n \Delta t)
$$

where the domain is divided into $M$ equal subintervals of length $\Delta x$, and the time step $\Delta t=t_{n+1}-t_{n}$ is also constant. When a finite-difference approximation is applied to the spatial derivative in (1), the following system of ordinary differential equations (ODE's) is obtained:

$$
\frac{d u}{d t}=\tilde{A} u
$$

where

$$
\tilde{A}=\frac{1}{\Delta x} A
$$

and

$$
u=\left[u_{1}, u_{2}, \ldots, u_{M}\right]^{T}
$$

The matrix $A$ is an $M \times M$ matrix that is typically banded. If the same difference operator is used at each node in the interior of the grid, then the entries are constant along the bands, except for the first and last few rows, i.e. $A$ is a quasi-Toeplitz matrix. The entries in these rows are determined by the numerical boundary schemes (NBS's).

When a time integration method is applied to the system of ODE's (3), the resulting difference equation can be written in the form

$$
v^{n+1}=C v^{n}
$$

where $v$ is a vector of length $M^{\prime}$ and $C$ is an $M^{\prime} \times M^{\prime}$ matrix. The vector $v$ contains the numerical solution at all of the time levels required by the differencing scheme. For example, for a two-step time-marching method, $M^{\prime}=2 M$ and

$$
v^{n}=\left[\begin{array}{c}
u^{n} \\
u^{n-1}
\end{array}\right]
$$

where $u$ is defined in (5). If a one-step time integration method is used, then $M^{\prime}=M$ and $v=u$. Note that the fully-discrete form (6) always exists, while the semi-discrete form (3) exists only if the spatial and temporal discretizations are performed separately. 


\section{B. Stability Definitions and Spectra}

As a result of the Lax equivalence theorem, Lax-Richtmyer stability is the most fundamental definition of stability. A difference approximation is Lax-Richtmyer stable if there exists a constant $K \geq 1$ (independent of $n$ ) such that, for an arbitrary initial condition $u^{0}$,

$$
\left\|u^{n}\right\| \leq K\left\|u^{0}\right\|
$$

for all $n \geq 0,0 \leq n \Delta t \leq T$ with $T$ fixed. A necessary and sufficient condition for Lax-Richtmyer stability is

$$
\left\|C^{n}\right\| \leq K
$$

for the same conditions as above, with $C$ defined in (6).

A necessary condition for stability is that the associated Cauchy problem (obtained by the assumption of periodic boundary conditions) is stable. The assumption of periodic boundary conditions produces a circulant matrix, sometimes called the circulant cousin of the quasi-Toeplitz matrix which includes the numerical boundary schemes. ${ }^{16}$ The Cauchy stability can be checked based on the spectrum of this circulant matrix. This tests the stability of the interior scheme independent of the boundary schemes.

Another useful concept, although neither necessary nor sufficient for Lax-Richtmyer stability, is asymptotic stability, which is the requirement that $\left\|C^{n}\right\|$ remain bounded as $n$ goes to infinity with fixed $M^{\prime}$. When consistent with the physics of the problem, the stronger condition that $\left\|C^{n}\right\|$ tend to zero as $n$ goes to infinity is a desirable property of a difference method. Since the solution of (1) tends to zero as $t$ goes to infinity when $U(0, t)=0$, the stronger condition is appropriate for discretizations of (1). This leads to the requirement that

$$
\rho(C)<1
$$

where $\rho(C)$ is the spectral radius of $C$, i.e. all of the eigenvalues of $C$ must lie within the unit circle in the complex plane.

For difference methods with an intermediate semi-discrete form, (10) is satisfied if all of the eigenvalues of $\Delta t \tilde{A}$ lie within the stable region of the time integration scheme. The ODE system (3) is termed inherently stable if the spectrum of $\tilde{A}$ lies entirely in the left half-plane. ${ }^{15}$ The solution of the ODE's then tends to zero as $t$ goes to infinity.

\section{Non-Normal Matrices and Pseudospectra}

If $C$ is a normal matrix, ${ }^{a}$ then its eigenvalue spectrum gives an accurate picture of its behaviour. The spectral radius is equal to the $\mathrm{L}_{2}$ norm, and the spectral radius condition (10) is sufficient for stability. If we assume that $C$ is nondefective, then the following relation holds between the matrix norms $\left\|C^{n}\right\|$ and the eigenvalues $\Lambda^{n}$ :

$$
\left\|C^{n}\right\|=\left\|X \Lambda^{n} X^{-1}\right\| \leq\|X\| \cdot\left\|X^{-1}\right\| \cdot\left\|\Lambda^{n}\right\|=\kappa(X)\left\|\Lambda^{n}\right\|
$$

where $X$ is the matrix of eigenvectors of $C, \Lambda$ the diagonal matrix of eigenvalues, and $\kappa(X)$ is the condition number of $X$. The relationship depends upon the condition number of the eigenvector matrix. For a normal matrix, the eigenvectors are orthogonal, and the condition number is unity.

For a non-normal matrix the eigenvectors can be far from orthogonal, and the condition number can be large. Therefore, even though the eigenvalue spectrum still describes the asymptotic behaviour accurately, $\left\|C^{n}\right\|$ can far exceed $\left\|\Lambda^{n}\right\|$. If the spectral radius of $C$ is less than unity, then $\left\|C^{n}\right\|$ must tend to zero as $n$ goes to infinity but can grow arbitrarily large for finite $n$. For nondefective $C$, we can write the solution to (6) as follows: ${ }^{15}$

$$
v^{n}=\sum_{m=1}^{M} c_{m}\left(\sigma_{m}\right)^{n} x_{m}
$$

where $\sigma_{m}$ and $x_{m}$ are the eigenvalues and eigenvectors of $C$. The coefficients $c_{m}$ are determined from the initial condition, i.e. the initial condition is decomposed into a linear combination of the eigenvectors. If $\rho(C)<1$, (12) shows that each eigenvector component of the solution must decay with increasing $n$. The norm of the solution can nevertheless increase because the eigenvectors are far from orthogonal. When an initial condition is represented as a linear combination of eigenvector components, the coefficients can be

a That is $C C^{*}=C^{*} C$. 


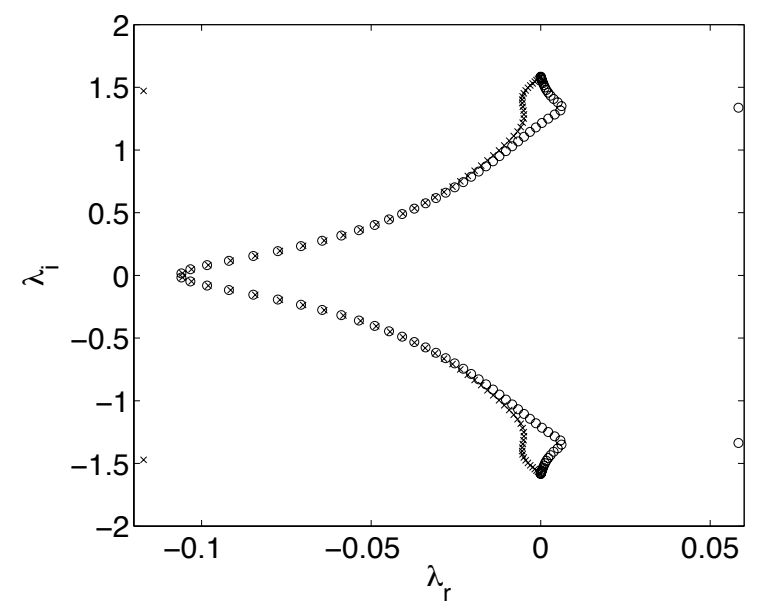

Figure 1. Eigenvalue spectra for sixth-order centered differences with conventional fifth-order inflow NBS (o) and stable fifth-order inflow NBS obtained with $\alpha=3 / 20, \beta=1 / 10(\mathbf{x}), M=100$.

very large. As the solution evolves, the cancellation needed to produce the initial condition is lost, and the norm of the solution can grow.

Pseudospectra provide a more accurate picture of the behaviour of non-normal matrices and operators. They have been applied to a wide range of applications, most extensively by Trefethen and co-workers. Rather than providing a list of references, other than the paper by Reddy and Trefethen ${ }^{12}$ and the book by Trefethen and Embree, ${ }^{13}$ we refer the reader to the Pseudospectra Gateway. ${ }^{17}$ This web site provides extensive information about pseudospectra, including definitions, theorems, a history, and a bibliography. It also provides software for computing pseudospectra, such as the EigTool for MATLAB. ${ }^{18}$

There are several definitions of pseudospectra. The most relevant here is the following definition in terms of eigenvalues: ${ }^{17}$

$$
\Lambda_{\epsilon}=\{z \in \mathrm{C}: z \in \Lambda(A+E) \text { for some } E \text { with }\|E\| \leq \epsilon\}
$$

Therefore an eigenvalue of a perturbed matrix $A+E$, where $E$ is a random matrix with norm $\epsilon$, is an $\epsilon$-pseudo-eigenvalue of $A$. If the $\epsilon$-pseudospectrum lies within $\epsilon$ of the spectrum, then the spectrum likely provides an accurate picture. If the deviation is significantly greater than $\epsilon$, then the pseudospectrum likely provides a more accurate predictor of the behaviour of $\|\exp (\tilde{A} t)\|$ at finite $t$ for the semi-discrete matrix $\tilde{A}$ in (3) and of $\left\|C^{n}\right\|$ at finite $n$ for the fully-discrete matrix $C$ in (6).

\section{Example 1: An Instability Related to the Inflow Boundary}

We consider a spatial discretization of the linear convection equation using sixth-order centered differences in the interior. In order to achieve sixth-order global accuracy, numerical boundary schemes of at least fifthorder are required. At the outflow boundary, fifth-order upwind and upwind-biased operators can be used. At the inflow boundary, fifth-order downwind-biased operators are a natural choice.

The spectrum of $A$ with $M=100$ is displayed in Figure 1. The criterion that all of the eigenvalues lie in the left half-plane is clearly violated. As $M$ tends to infinity, the eigenvalue spectrum of a quasi-Toeplitz matrix can be partitioned into a boundary-condition independent spectrum and a boundary-condition dependent spectrum. ${ }^{16}$ The boundary condition independent spectrum is the spectrum of the Toeplitz matrix associated with the auxiliary Dirichlet problem found by replacing all boundary conditions by homogeneous Dirichlet boundary conditions. In the asymptotic limit, the spectrum of the auxiliary Dirichlet matrix is bounded by the spectrum of the circulant cousin associated with the Cauchy problem. If the Cauchy problem is stable, any instabilities must thus be associated with the boundary-condition dependent spectrum, which is independent of the matrix size, and are related to the NBS's. ${ }^{4}$ In our example, there exist two boundary-condition dependent eigenvalues that lie in the right half-plane. 


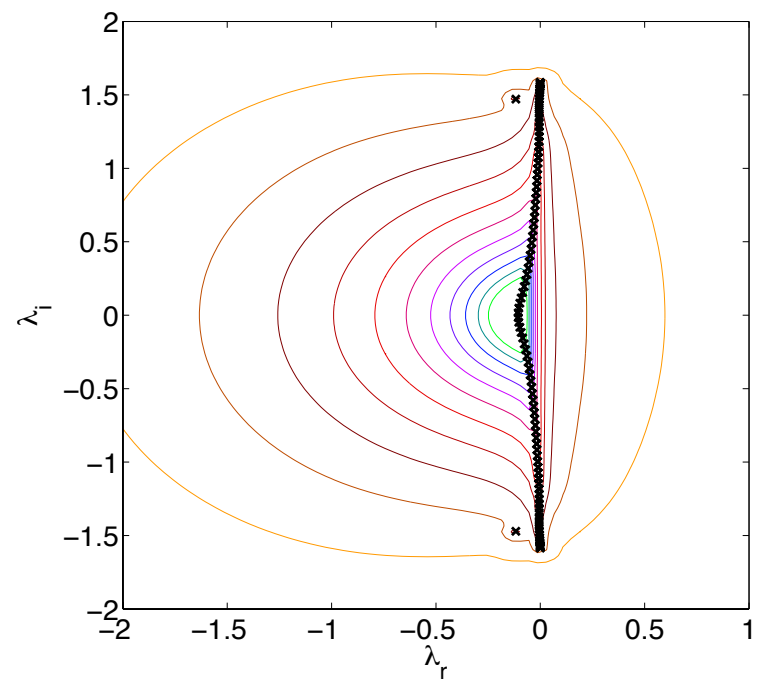

Figure 2. Spectrum (x) and pseudospectra for example 2 with $M=100$.

\section{Example 2: Stable Inflow Boundary Schemes}

Next we consider the modified inflow NBS developed by Zingg et al. ${ }^{6,19,20}$ The stencil of the operators at the two grid nodes nearest the inflow boundary is increased by one element beyond that needed for fifth-order accuracy. This results in the following two-parameter family of fifth-order inflow NBS's:

$$
\begin{aligned}
\left(\delta_{x} u\right)_{1}= & \frac{1}{60 \Delta x}\left[(-12+60 \alpha) u_{0}+(-65-360 \alpha) u_{1}+(120+900 \alpha) u_{2}\right. \\
& \left.+(-60-1200 \alpha) u_{3}+(20+900 \alpha) u_{4}+(-3-360 \alpha) u_{5}+60 \alpha u_{6}\right] \\
\left(\delta_{x} u\right)_{2}= & \frac{1}{60 \Delta x}\left[(3+60 \beta) u_{0}+(-30-360 \beta) u_{1}+(-20+900 \beta) u_{2}\right. \\
& \left.+(60-1200 \beta) u_{3}+(-15+900 \beta) u_{4}+(2-360 \beta) u_{5}+60 \beta u_{6}\right]
\end{aligned}
$$

The spectrum obtained with $\alpha=3 / 20, \beta=1 / 10$ is displayed in Figure 1 . The entire spectrum lies in the left half-plane. There are still two boundary-condition dependent eigenvalues, but they now lie well in the left half-plane. Therefore, we have both Cauchy stability and asymptotic stability. Pseudospectra are shown in Figure 2. There are no bulges characteristic of instability. Hence we conclude that the scheme is Lax-Richtmyer stable.

\section{Example 3: A Mild Instability Related to the Interior Difference Operator}

Next we consider a full discretization with a mild instability caused by the interior difference scheme. The spatial operator is given by sixth-order centered differences plus a symmetric operator given by

$$
\left(\delta_{x}^{s} U\right)_{j}=\frac{1}{\Delta x}\left[d_{3}\left(U_{j+3}+U_{j-3}\right)+d_{2}\left(U_{j+2}+U_{j-2}\right)+d_{1}\left(U_{j+1}+U_{j-1}\right)+d_{0} U_{j}\right]
$$

with $d_{0}=0.1, d_{1}=-0.0804523, d_{2}=0.0403545$, and $d_{3}=-0.00990220$. When applied to

$$
\frac{d U}{d t}=f(U, t)
$$

the six-stage time-marching method is given by: ${ }^{19}$ 


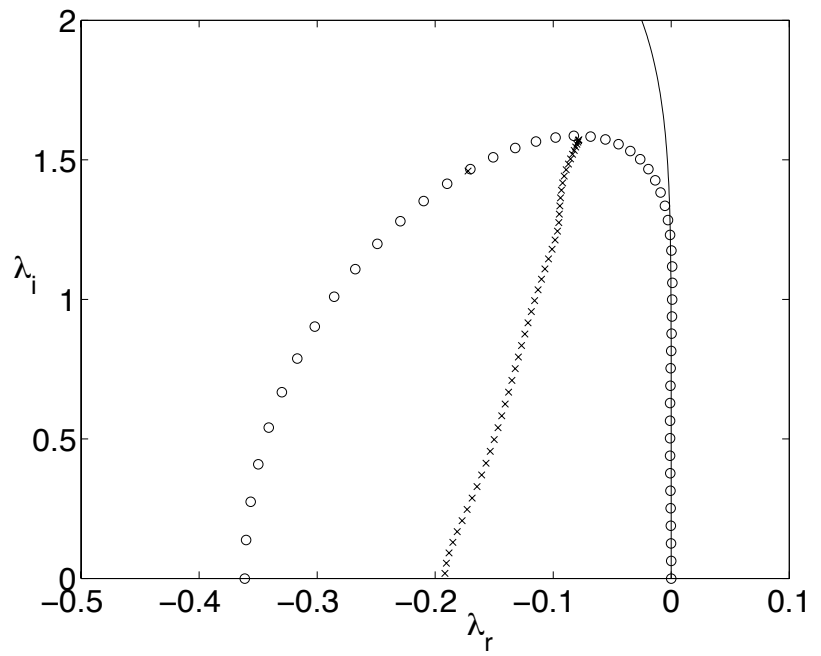

Figure 3. Spectra of $A$ with periodic boundary conditions (o) and NBS's (x) and the stability contour of the time integration method $(18)(-)$.

$$
\begin{aligned}
U_{n+\alpha_{1}}^{(1)} & =U_{n}+\Delta t \alpha_{1} f_{n} \\
U_{n+\alpha_{2}}^{(2)} & =U_{n}+\Delta t \alpha_{2} f_{n+\alpha_{1}}^{(1)} \\
U_{n+\alpha_{3}}^{(3)} & =U_{n}+\Delta t \alpha_{3} f_{n+\alpha_{2}}^{(2)} \\
U_{n+\alpha_{4}}^{(4)} & =U_{n}+\Delta t \alpha_{4} f_{n+\alpha_{3}}^{(3)} \\
U_{n+\alpha_{5}}^{(5)} & =U_{n}+\Delta t \alpha_{5} f_{n+\alpha_{4}}^{(4)} \\
U_{n+1} & =U_{n}+\Delta t f_{n+\alpha_{5}}^{(5)}
\end{aligned}
$$

where $U_{n}=U\left(t_{n}\right)$, and

$$
f_{n+\alpha}^{(k)}=f\left(U_{n+\alpha}^{(k)}, t_{n}+\alpha h\right)
$$

with $\alpha_{1}=1 / 6, \alpha_{2}=1 / 5, \alpha_{3}=1 / 4, \alpha_{4}=1 / 3, \alpha_{5}=1 / 2$.

The NBS at the inflow boundary is as given in (14) and (15) with $\alpha=3 / 20, \beta=1 / 10$. At the outflow boundary, fifth-order upwind and upwind-biased operators are used. The matrix entries are given in the Appendix. Figure 3 shows the stability contour of the time-marching method with a solid line. The eigenvalues of the associated circulant matrix are shown by the symbol "o". Some lie just outside the stable region of the time-marching method (near the imaginary axis between 0.8 and 1.2). The eigenvalue spectrum of the spatial operator matrix with NBS's and $M=100$ is shown by the symbol "x", demonstrating the asymptotic stability of the method for this value of $M$.

Figure 4 shows the pseudospectra for $M=5000$. The bulge in the pseudospectra clearly reveals the instability. In Zingg ${ }^{14}$ the matrix used was too small to reveal such a mild instability. The pseudospectra are clearly helpful in revealing the instability in this case, and the spectrum is not. However, the instability is also easily seen by consideration of the spectrum of the associated circulant operator, which can be found analytically.

\section{Example 4: An Instability Related to the Outflow Boundary: Explicit Scheme}

Next we consider an example from Trefethen and Embree. ${ }^{13}$ In order to maintain consistency with their notation, we consider the linear convection equation with the direction of propagation reversed, i.e.

$$
\frac{\partial U}{\partial t}=\frac{\partial U}{\partial x}
$$




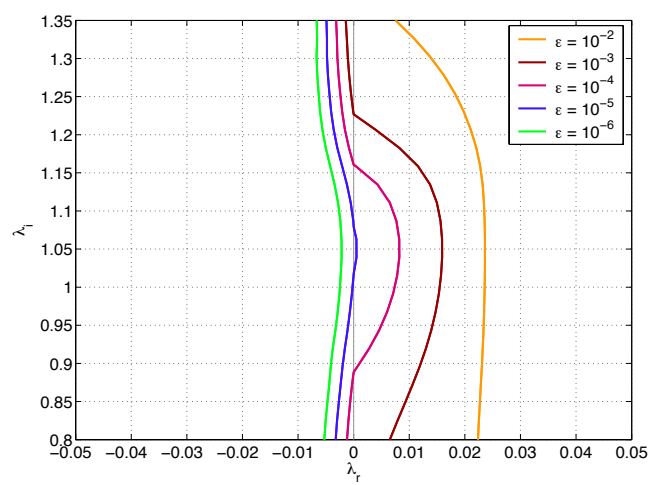

Figure 4. Pseudospectra for example 3 with $M=5000$.

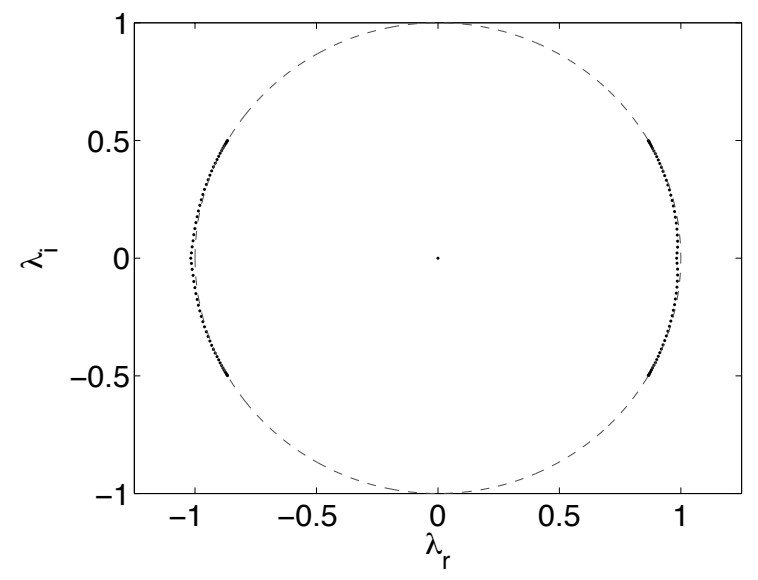

Figure 5. Eigenvalue spectrum for example 4 with $\Delta t=\Delta x / 2$ and $M=60$.

Therefore, the left boundary becomes the outflow boundary, and the right boundary is the inflow boundary. Second-order centered differencing is used together with the leapfrog time-marching method, giving the following fully-discrete scheme:

$$
\frac{u_{j}^{n+1}-u_{j}^{n-1}}{2 \Delta t}=\frac{u_{j+1}^{n}-u_{j-1}^{n}}{2 \Delta x}
$$

The boundary scheme at the outflow boundary is

$$
u_{0}^{n+1}=u_{1}^{n+1}
$$

and at the inflow boundary, $u_{N}^{n+1}=0$. This is a two-step method, so $M^{\prime}=2 M$.

The eigenvalue spectrum of $C$ for $M=60$ with a Courant number of $1 / 2$ is displayed in Figure 5. Since the spectral radius exceeds unity, the scheme is asymptotically unstable. Pseudospectra are shown in Figure 6 showing a bulge characteristic of an instability precisely in the vicinity of the eigenvalue of largest magnitude. Figure 7 shows the singular vector associated with the maximum singular value of $C^{60}$ and also the product of $C^{60}$ and this singular vector. Note that $v$ includes both $u^{n}$ and $u^{n-1}$, as in (7). For this example $\left\|C^{60}\right\|$ is 39.140966 . If the singular vector were prescribed as the initial condition, then the solution norm would grow by this factor after 60 time steps. The nature of the singular vector clearly points to the outflow (left) boundary as the cause of the instability. Both the spectrum and the pseudospectra are effective in revealing the instability for this example. 


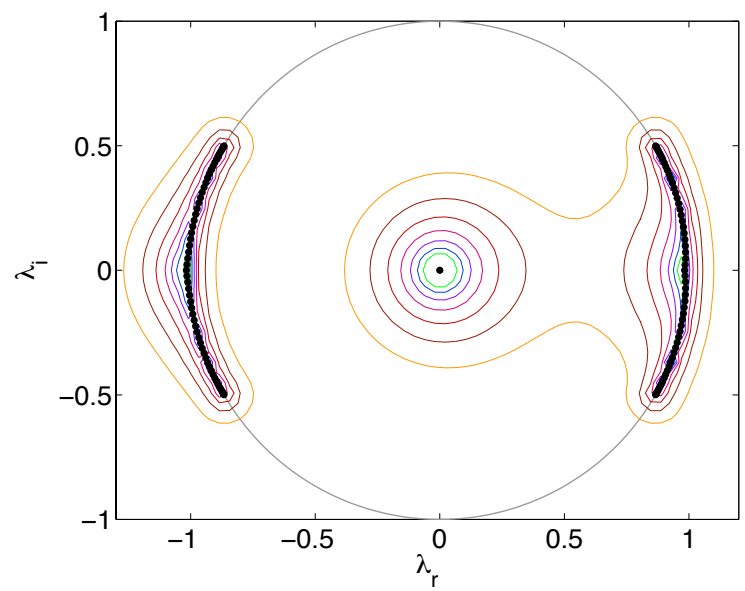

Figure 6. Pseudospectra for example 4 with $\Delta t=\Delta x / 2$ and $M=60$.

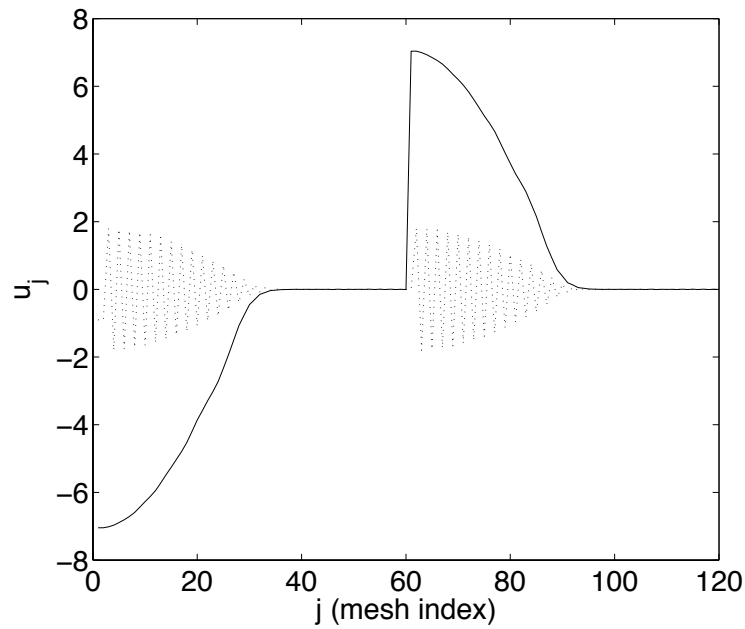

Figure 7. Singular vector of $C^{60}(\cdots)$ for example 4 and the product of $C^{60}$ and this singular vector (-). Note that the singular vector has been amplified by a factor of ten.

\section{Example 5: An Instability Related to the Outflow Boundary: Implicit Scheme}

This example is an unstable implicit scheme from Trefethen and Embree. ${ }^{13}$ The spatial discretization is again second-order centered differences, but the time-marching method is the trapezoidal method. ${ }^{15}$ The fully-discrete scheme is given by

$$
\frac{u_{j}^{n+1}-u_{j}^{n}}{\Delta t}=\frac{u_{j+1}^{n}-u_{j-1}^{n}}{4 \Delta x}+\frac{u_{j+1}^{n+1}-u_{j-1}^{n+1}}{4 \Delta x}
$$

The boundary scheme at the outflow boundary is

$$
u_{0}^{n+1}=u_{2}^{n}
$$

and at the inflow boundary, $u_{N}^{n+1}=0$. This is a one-step scheme, so $M^{\prime}=M$. 


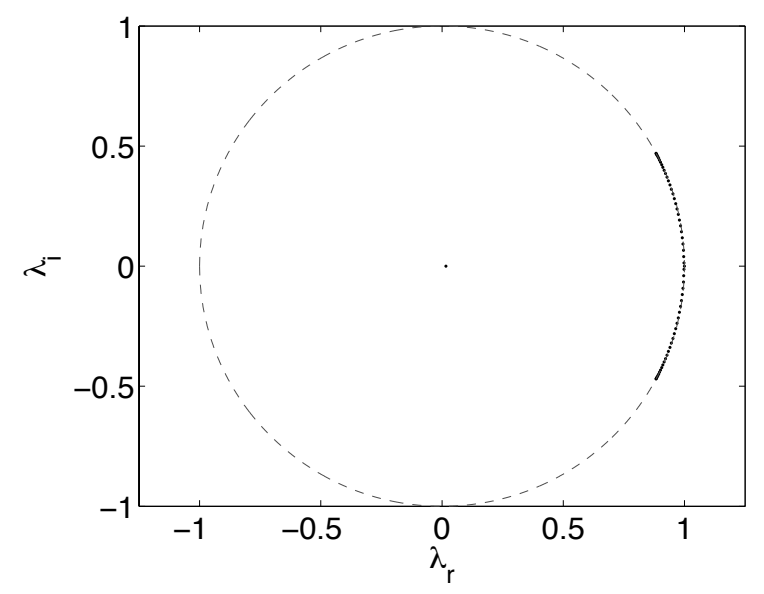

Figure 8. Eigenvalue spectrum for example 5 with $\Delta t=\Delta x / 2$ and $M=60$.

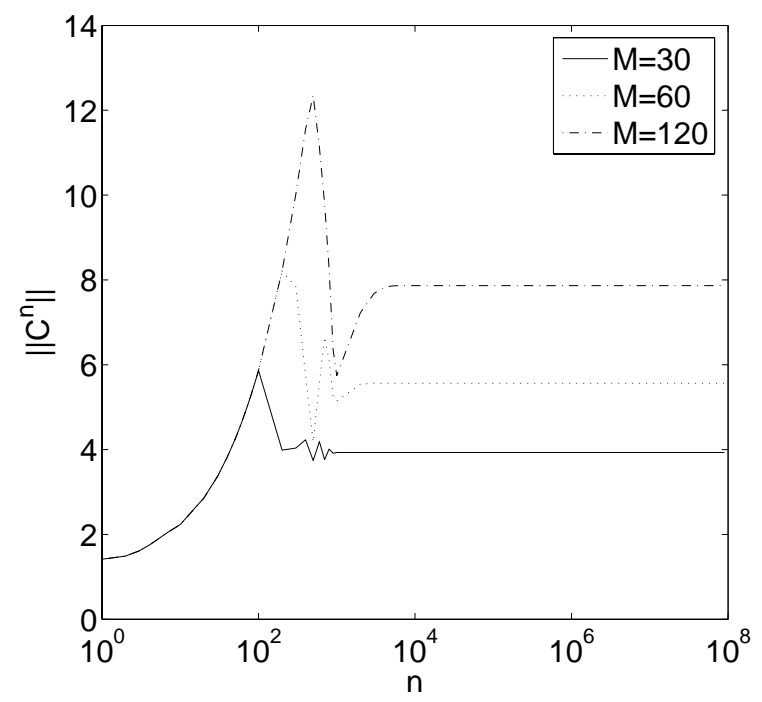

Figure 9. $\left\|C^{n}\right\|$ vs. $n$ for example 5 with $\Delta t=\Delta x / 2$ and $M=30,60,120$.

Figure 8 displays the eigenvalue spectrum with a Courant number of $1 / 2$ and $M=60$. The spectral radius is equal to unity. This discretization produces an eigenvalue equal to unity independent of $M$. The corresponding eigenvector has the form $\left[\begin{array}{llllll}0 & 1 & 0 & 1 \ldots\end{array}\right]^{T}$ for $M$ even and $\left[\begin{array}{llllll}1 & 0 & 1 & 0 & \ldots\end{array}\right]^{T}$ for $M$ odd. Matrix norms $\left\|C^{n}\right\|$ are plotted in Figures 9 and 10 for even and odd $M$, respectively. When $M$ is even, $\left\|C^{n}\right\|$ has a peak and then approaches its asymptotic value, which increases with $M$. For odd $M$, the asymptotic value is much higher and is approached monotonically. The instability is clearly evident from the bulge in the pseudospectra shown in Figure 11. The singular vector corresponding to the maximum singular value of $C^{M}$ is shown in Figure 12 for $M=60$ as well as the solution after $M$ time steps when the singular vector is given as the initial condition. The $\mathrm{L}_{2}$ norm of $C^{60}$ is 4.608965 . The singular vector shows that the instability is associated with the NBS at the outflow (left) boundary.

This example owes its particular behaviour to the fact that the spectral radius of $C$ is equal to unity for 


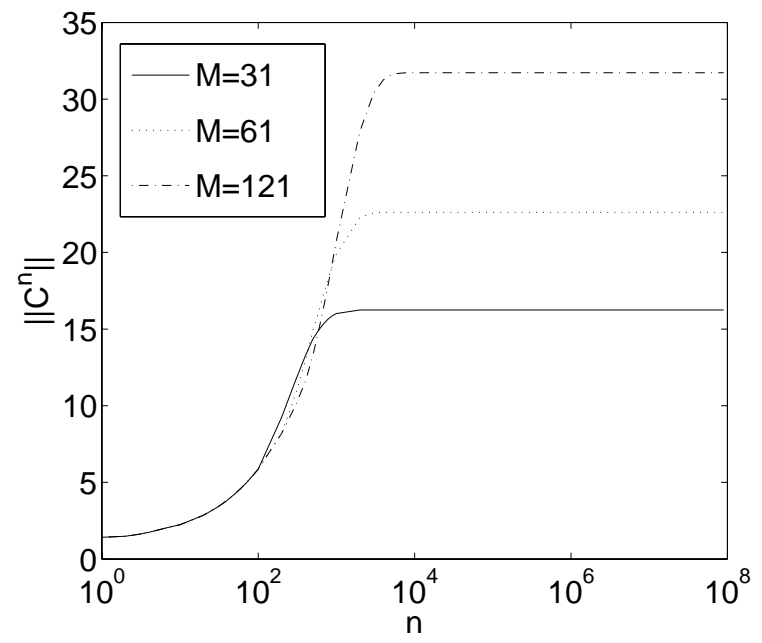

Figure 10. $\left\|C^{n}\right\|$ vs. $n$ for example 5 with $\Delta t=\Delta x / 2$ and $M=31,61,121$.

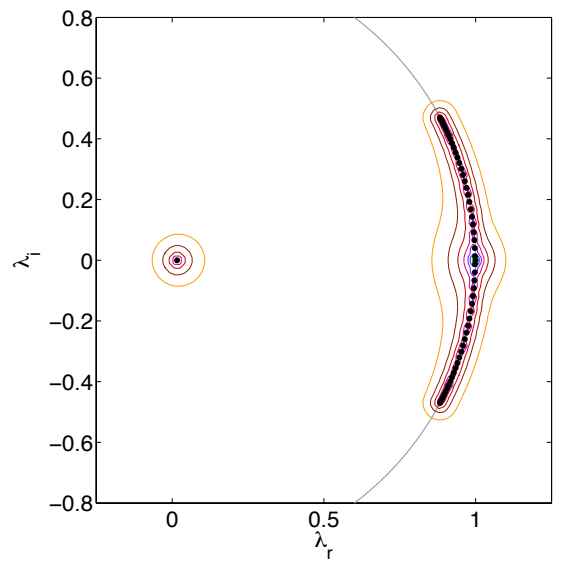

Figure 11. Pseudospectra for example 5 with $\Delta t=\Delta x / 2$ and $M=60$.

all $M$. Consequently, $\lim _{n \rightarrow \infty}\left\|C^{n}\right\|$ is finite. For $M=5$ and a Courant number of $1 / 2$ we obtain:

$$
\lim _{n \rightarrow \infty} C^{n}=\left[\begin{array}{ccccc}
1 / 2 & -4 & 1 / 2 & 0 & 0 \\
0 & 0 & 0 & 0 & 0 \\
1 / 2 & -4 & 1 / 2 & 0 & 0 \\
0 & 0 & 0 & 0 & 0 \\
1 / 2 & -4 & 1 / 2 & 0 & 0
\end{array}\right]
$$

This matrix has an $\mathrm{L}_{2}$ norm of 7.0356. The corresponding singular vector is $\left[\begin{array}{lllll}-0.1231 & 0.9847 & 0.1231 & 0 & 0\end{array}\right]^{T}$, which is quite close to the singular vector for $M=60$ shown in Figure 12. The product of the matrix given in $(24)$ and this singular vector is $\left[\begin{array}{lllll}-4.062 & 0 & -4.062 & 0 & -4.062\end{array}\right]^{T}$. These results can be extended to find that, for odd $M$ :

$$
\lim _{n \rightarrow \infty}\left\|C^{n}\right\|=4.062 \sqrt{\frac{M+1}{2}}
$$




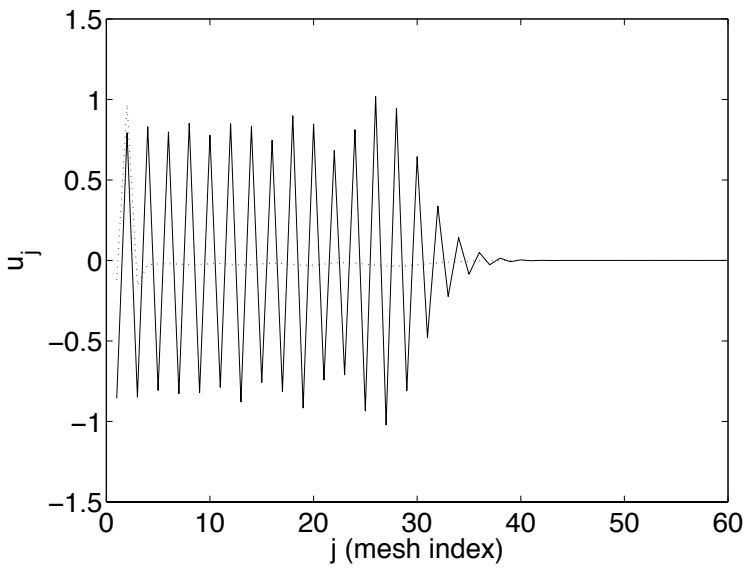

Figure 12. Singular vector of $C^{60}(\cdots)$ for example 5 and the product of $C^{60}$ and this singular vector $(-)$.

Similarly, for $M=6$ we obtain:

$$
\lim _{n \rightarrow \infty} C^{n}=\left[\begin{array}{cccccc}
0 & 0 & 0 & 0 & 0 & 0 \\
1 / 8 & 1 & -1 / 8 & 0 & 0 & 0 \\
0 & 0 & 0 & 0 & 0 & 0 \\
1 / 8 & 1 & -1 / 8 & 0 & 0 & 0 \\
0 & 0 & 0 & 0 & 0 & 0 \\
1 / 8 & 1 & -1 / 8 & 0 & 0 & 0
\end{array}\right]
$$

which has a norm of 1.7589 , one quarter of the norm of the matrix with $M=5$ given in (24). The nonzero entries in the singular vector are identical to those for $M=5$ given above, and the product is $\left[\begin{array}{lllllll}0 & 1.0155 & 0 & 1.0155 & 0 & 1.0155\end{array}\right]^{T}$. Thus for even $M$ we find

$$
\lim _{n \rightarrow \infty}\left\|C^{n}\right\|=1.0155 \sqrt{\frac{M}{2}}
$$

These results are consistent with the discussion in Trefethen and Embree ${ }^{13}$ and the results shown in Figures 9 and 10.

This example is interesting in that the pseudospectra identify a subtle instability. The spectrum also violates our tight spectral radius condition (10).

\section{Discussion}

We return now to example 2, in which a stable inflow boundary scheme is given for the linear convection equation with sixth-order centered differences in the interior. This was found by increasing the stencil of the numerical boundary scheme one node beyond the stencil needed to achieve fifth-order accuracy (and hence sixth-order global accuracy). The resulting free parameter values, $\alpha=3 / 20, \beta=1 / 10$, in (14) and (15) were selected by trial and error to obtain the eigenvalue spectrum displayed in Fig. 1. It would be preferable to determine $\alpha$ and $\beta$ in a more systematic manner by solving a minimization problem. The question is: What should we minimize?

A natural choice is the spectral abscissa, the largest real part of all of the eigenvalues of a matrix. Minimizing the spectral abscissa with $M=100$ leads to $\alpha=0.13843, \beta=0.06657$. This reduces the spectral abscissa from $-4.8767 \times 10^{-5}$ (for the baseline scheme with $\alpha=3 / 20, \beta=1 / 10$ ) to $-4.328 \times 10^{-4}$. As $M$ is increased the values of $\alpha$ and $\beta$ that minimize the spectral abscissa change, but not dramatically. ${ }^{\mathrm{b}}$ The resulting spectra are compared in Figure 13. The real part of quite a few eigenvalues has increased near

\footnotetext{
${ }^{\mathrm{b}}$ For example, with $M=1000$, we obtain $\alpha=0.13509, \alpha=0.06376$.
} 


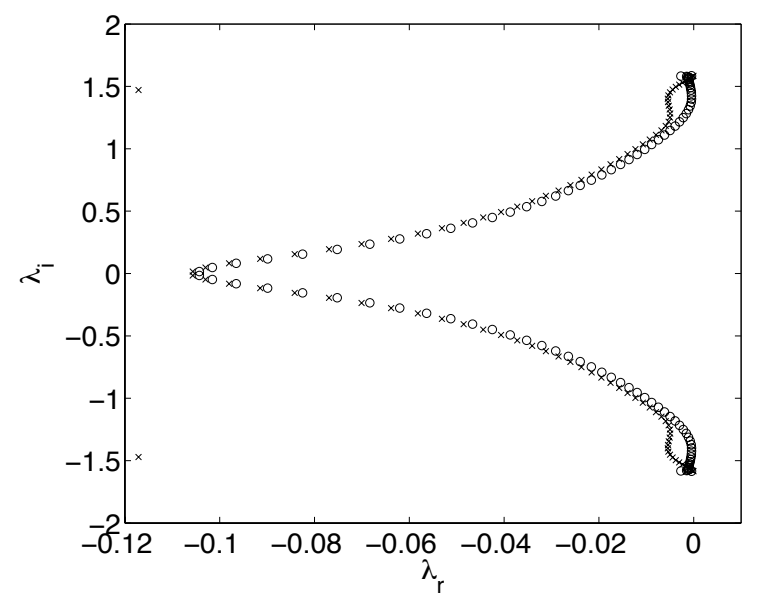

Figure 13. Spectra for example 2 with $\alpha=3 / 20$ and $\beta=1 / 10(\mathrm{x})$ and the scheme with minimum spectral abscissa (o), $M=60$.

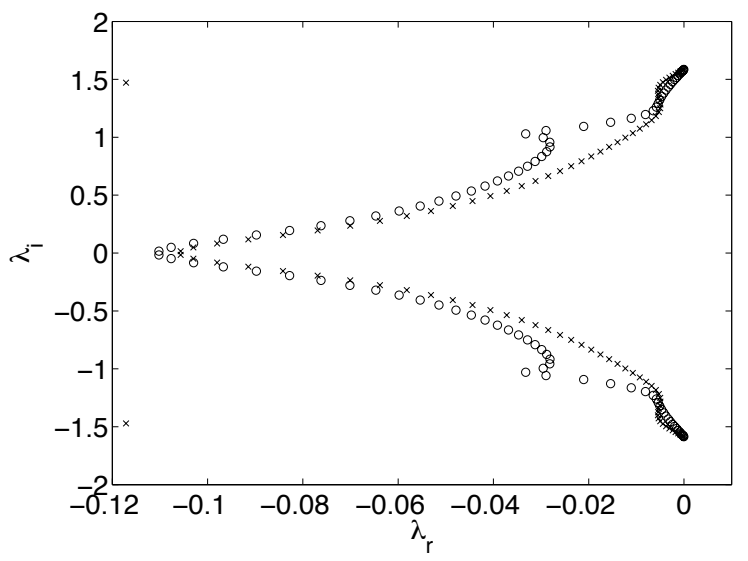

Figure 14. Spectra for example 2 with $\alpha=3 / 20$ and $\beta=1 / 10(\mathbf{x})$ and the scheme with minimum $\int_{0}^{\tau}\|\exp (A t)\| \mathrm{d} t$ (o), $M=60$.

the imaginary axis. Hence it is not clear that this numerical boundary scheme is preferable to the baseline scheme despite the reduction in the spectral abscissa.

Another possibility is to choose $\alpha$ and $\beta$ to minimize $\int_{0}^{\tau}\|\exp (A t)\| \mathrm{d} t$ for some value of $\tau$. For large $\tau$ this gives $\alpha=0.033, \beta=0.0729$. The spectrum for this scheme is displayed in Figure 14. The real part of a significant number of eigenvalues has been reduced. Hence the stability properties resulting from these values of $\alpha$ and $\beta$ could be superior when applied to nonlinear problems or curvilinear meshes.

Svard et al. ${ }^{22}$ have recently examined the NBS given in example 2 coupled with sixth-order centered differences applied to a more challenging problem, two coupled advection equations where no energy escapes the system. In this context they found the NBS to produce eigenvalues with positive real parts, while the spectrum of their summation-by-parts operator lies strictly in the left half-plane. In future work, we intend to examine this further.

\section{Conclusions}

The examples have shown that pseudospectra are a useful means for detecting instabilities of finitedifference methods. In each case the instability is also revealed by violation of one of the following two 
conditions:

$$
\rho(C)<1
$$

or

$$
\rho\left(C_{p}\right) \leq 1
$$

where $C_{p}$, the circulant cousin of $C$, is obtained from the assumption of periodic boundary conditions. The singular vector associated with the maximum singular value of $C^{M}$ is also seen as a good indicator of the cause of the instability.

\section{Appendix}

This Appendix gives the entries of the matrix $A$ for the unstable scheme. The fully-discrete matrix is found from:

$$
C=I+N_{\mathrm{CFL}} A+\left(N_{\mathrm{CFL}} A\right)^{2} / 2+\left(N_{\mathrm{CFL}} A\right)^{3} / 6+\left(N_{\mathrm{CFL}} A\right)^{4} / 24+\left(N_{\mathrm{CFL}} A\right)^{5} / 120+\left(N_{\mathrm{CFL}} A\right)^{6} / 720
$$

where $N_{\text {CFL }}$ is the CFL number $\Delta t / \Delta x$. The nonzero entries in the first row of $A$ are (to eight decimal places): $1.98333333,-4.25,4.0,-2.58333333,0.95,-0.15$. The nonzero entries in the second row are: $1.1,-1.16666667,1.0,-1.25,0.56666667,-0.1$. These two rows correspond to the NBS given in eqs. 14 and 15 with $\alpha=3 / 20, \beta=1 / 10$. The nonzero entries in the third row are: -0.19035450 , $0.83045230,-0.1,-0.66954770,0.10964550,-0.00676447$, while those in the fourth row are: 0.02656887 , $-0.19035450,0.83045230,-0.1,-0.66954770,0.10964550,-0.00676447$, corresponding to the interior operator. The nonzero entries in the final three rows, which correspond to fifth-order operators, are: 0.03333333 , $-0.23094130,0.93191930,-0.23528933,-0.56808070,0.06905870$ for the third-last row, -0.06905870 , $0.44768553,-1.26682180,2.31309330,-1.27116980,-0.15372850$ for the second-last row, and 0.15372850 , $-0.99142970,2.75361300,-4.34139180,4.61902080,-2.19352080$ for the last row.

\section{Acknowledgments}

The funding of the first author by the Natural Sciences and Engineering Research Council of Canada and the Canada Research Chairs program is gratefully acknowledged. Thanks to Song Yue for Figure 4.

\section{References}

\footnotetext{
${ }^{1}$ Gustafsson, B., "The Convergence Rate for Difference Approximations to Mixed Initial Boundary Value Problems," Math. Comp., Vol. 49, No. 130, pp. 396-406, 1975.

${ }^{2}$ Godunov, S., and Ryabenkii, V., "Special Stability Criteria of Boundary-Value Problems for Non-Self-Adjoint Difference Equations," Russ. Math. Surv., Vol. 18, pp. 1-12, 1963.

${ }^{3}$ Gustafsson, B., Kreiss, H.O., Sundstrom, A., "Stability Theory of Difference Approximations for Mixed Initial Boundary Value Problems II," Math. Comp., Vol. 26, pp. 649-686, 1972.

${ }^{4}$ Warming, R.F., and Beam, R.M., "Lecture Notes on Stability and Accuracy of Difference Approximations for InitialBoundary-Value Problems," NASA Ames Research Center, Feb. 1993.

${ }^{5}$ Carpenter, M.H., Gottlieb, D., and Abarbanel, S., "Stable and Accurate Boundary Treatments for Compact, High-Order Finite-Difference Schemes," Applied Numerical Mathematics, Vol. 12, pp. 55-87, 1993.

${ }^{6}$ Zingg, D.W., and Lomax, H., "On the Eigensystems Associated with Numerical Boundary Schemes for Hyperbolic Equations," in Numerical Methods for Fluid Dynamics, M.J. Baines and K.W. Morton, eds., Clarendon Press, Oxford, pp. 471-480, 1993.

${ }^{7}$ Kreiss, H.-O., and Scherer, G., "Finite Element and Finite Difference Methods for Hyperbolic Partial Differential Equations," in Mathematical Aspects of Finite Elements in Partial Differential Equations, Academic Press, New York, 1974.

${ }^{8}$ Strand, B., "Summation by Parts for Finite Difference Approximations for d/dx," J. Comp. Phys., Vol. 110, pp. 47-67, 1994.

${ }^{9}$ Olsson, P., "Summation by Parts, Projections, and Stability I," Math. Comp., Vol. 64, pp. 1035-1065, 1995.

${ }^{10}$ Mattsson, K., and Nordstrom, J., "Boundary Procedures for Summation-By-Parts Operators," J. of Scientific Computing, $18,2003$.

${ }^{11}$ Carpenter, M.H., Gottlieb, D., and Abarbanel, S., "Time-Stable Boundary Conditions for Finite-Difference Schemes Solving Hyperbolic Systems: Methodology and Application to High-Order Compact Schemes," J. Comp. Phys., Vol. 111, pp. 220-236, 1994.

${ }^{12}$ Reddy, S.C., and Trefethen, L.N., "Stability of the Method of Lines," Numer. Math., Vol. 62, pp. 235-267, 1992.

${ }^{13}$ Trefethen, L.N., and Embree, M., Spectra and Pseudospectra: The Behavior of Non-Normal Matrices and Operators, book in preparation.
} 
${ }^{14}$ Zingg, D.W., "Aspects of Linear Stability Analysis for Higher-Order Finite-Difference Methods," AIAA Paper 97-1939, June 1997.

${ }^{15}$ Lomax, H., Pulliam, T.H., and Zingg, D.W., Fundamentals of Computational Fluid Dynamics, Springer-Verlag, 2001.

${ }^{16}$ Beam, R.M., and Warming, R.F., "The Asymptotic Spectra of Banded Toeplitz and Quasi-Toeplitz Matrices," SIAM J. on Scientific Computing, Vol. 14, No. 4, pp. 971-1006, 1993.

${ }^{17}$ Pseudospectra Gateway, http://web.comlab.ox.ac.uk/projects/pseudospectra/.

${ }^{18}$ Wright, T.G., and Trefethen, L.N., "Large-scale Computation of Pseudospectra using ARPACK," SIAM J. Sci. Comp., Vol. 23, pp. 591-605, 2001.

${ }^{19}$ Zingg, D.W., Lomax, H., and Jurgens, H., "High-Accuracy Finite-Difference Schemes for Linear Wave Propagation," SIAM J. on Scientific Computing, Vol. 17, No. 2, March 1996, pp. 328-346.

${ }^{20}$ Jurgens, H.M., and Zingg, D.W., "Numerical Solution of the Time-Domain Maxwell Equations Using High-Accuracy Finite-Difference Methods," SIAM J. Sci. Comput., Vol. 22, No. 5, pp. 1675-1696, 2000.

${ }^{21}$ Beam, R.M., Warming, R.F., and Yee, H.C., "Stability Analysis of Numerical Boundary Conditions and Implicit Difference Approximations for Hyperbolic Equations," J. Comp. Phys., Vol. 48, pp. 200-222, 1982.

${ }^{22}$ Svard, M., Mattsson, K., and Nordstrom, J., "Steady State Computations Using Summation-by Parts Operators," to appear in Journal of Scientific Computing. 